\title{
Intervalo de tempo entre observações para avaliação do comportamento ingestivo de tourinhos em confinamento
}

\author{
Intervals between observations for ingestive \\ behavior evaluation of young bulls in feedlot
}

\author{
Jair de Araújo Marques ${ }^{1 *}$; Andréa Pereira Pinto²; José Jorge dos Santos Abrahão²; \\ Willian Gonçalves do Nascimento ${ }^{2}$
}

\begin{abstract}
Resumo
Objetivou-se com o experimento estudar intervalos de tempo para avaliação do comportamento ingestivo de tourinhos confinados. Foram utilizados 36 animais, alojados em baias individuais, em delineamento inteiramente casualizado, com três tratamentos (silagem de sorgo com 1,0 \% de concentrado, cana-deaçúcar com 1,0\% de concentrado e com 1,2\% de concentrado). Foram comparadas cinco escalas de intervalos de observações. Avaliou-se o tempo e freqüência de alimentação, ruminação e ócio, sendo que destes dois últimos itens calculou-se a percentagem do tempo que os animais permaneciam deitados, ingestão de matéria seca e de fibra em detergente neutro, tempo de mastigação total e eficiência de alimentação e de ruminação da matéria seca e da fibra em detergente neutro. Não se observou diferença significativa $(\mathrm{P}>0,05)$ entre as escalas de tempo analisadas para as variáveis relacionadas a tempo e eficiência. A percentagem de tempo que os animais permaneceram ruminando deitado ficou em torno de $91 \%$. As freqüências de ingestão, ruminação e ócio diferiram $(\mathrm{P}<0,05)$ para todas as escalas de tempo avaliadas. Concluiu-se que o estudo do comportamento ingestivo de ruminantes pode ser efetuado com escalas de até 30 minutos de intervalo quando não se avalia a freqüência.

Palavras-chave: Cana-de-açúcar, eficiência, ingestão, ócio, ruminação, silagem de sorgo
\end{abstract}

\begin{abstract}
The objective of this experiment was to study the intervals of time for observing the ingestive behavior of 36 young crossbred bulls in feedlot. The animals were housed in individual stalls, in a completely randomized design, with three treatments (sorghum silage with $1.0 \%$ concentrate, sugar cane with 1.0 $\%$ concentrate and sugar cane with $1.2 \%$ concentrate). Five different intervals between observations were studied. The evaluated parameters were: feeding, rumination and idle, time and frequency, dry matter and neutral detergent fiber intake, total chewing time, feeding and rumination efficiency of dry matter and neutral detergent fiber, as well as, the percentage of the time in that the animals were laying during rumination or idle. The intervals of time did not differ $(\mathrm{P}>0.05)$ for analyzed variable of the time and efficiency. The percentage of time in that the animals were laid while ruminating, was $91 \%$. The frequencies of feeding, rumination and idle, differed $(\mathrm{P}<0.05)$ among all intervals. It can be concluded that the evaluation of ingestive behavior can be carried out at 30 minute intervals when did not study the frequency.
\end{abstract}

Key words: Efficiency, feed intake, idle, rumination, sorghum silage, sugar cane

1 Professor Adjunto de Bovinocultura de Corte do CCAAB da UFRB, Cruz das Almas, BAPesquisador do convênio IAPAR/EMA. CEP 44380-000. E-mail: jmarques@ufrb.edu.br

2 Pesquisadores do Instituto Agronômico do Paraná - Iapar, Estação Experimental de Paranavaí.

* Autor para correspondência 


\section{Introdução}

O manejo nutricional adequado dos animais na propriedade depende de vários fatores, dentre os quais o conhecimento do comportamento ingestivo dos animais, relacionado com a característica do alimento fornecido.

O estudo do comportamento animal é uma ferramenta de grande importância, principalmente, para animais mantidos em regime de confinamento (DAMASCENO; BACCARI JÚNIOR; TARGA, 1999). Portanto, com a necessidade do entendimento do comportamento ingestivo dos ruminantes, temse investido em pesquisas que forneçam dados que permitam proporcionar aos animais um manejo nutricional adequado, bem como a influência do comportamento ingestivo sobre o consumo de alimentos (SILVA et al., 2005).

Existem várias técnicas de registro de dados, como observações visuais, registros semi-automáticos e automáticos (BÜRGER et al., 2000). Entretanto, para se obter alta confiabilidade nas informações, faz-se necessário estabelecer a metodologia a ser utilizada, sendo um fator muito importante, o intervalo de tempo entre as observações, uma vez que a observação visual contínua dos animais é um processo que necessita de muita mão-de-obra, tornando-se impraticável quando se deseja observar um número elevado de animais (SILVA et al., 2005).

A escolha da escala de intervalo entre as observações, pode comprometer os resultados das avaliações, portanto, para que se obter resultados de alta confiabilidade torna-se necessário estabelecer a metodologia mais adequada a ser empregada de acordo com o que se pretende avaliar.

Este trabalho foi realizado com o objetivo de estudar cinco intervalos de tempo para avaliação do comportamento ingestivo de tourinhos confinados, recebendo dietas completas compostas de cana-deaçúcar ou silagem de sorgo e concentrado.

\section{Material e Métodos}

O experimento foi realizado no setor de confinamento, do Instituto Agronômico do Paraná na Unidade Regional de Pesquisa de Paranavaí. Foram utilizados 36 tourinhos mestiços, com idade média de $18 \pm 2$ meses, com peso médio de $398 \pm 10 \mathrm{~kg}$, oriundos do programa de cruzamento da Estação.

Os animais foram alojados em baias individuais, com acesso a cocho de alimentação e água, distribuídos em três tratamentos (cana-de-açúcar picada $+1,0 \%$ de concentrado, cana-de-açúcar picada $+1,2 \%$ de concentrado e silagem de sorgo $+1,0 \%$ de concentrado), sendo a porcentagem de concentrado calculada com base no peso vivo (PV), em matéria seca, reajustado a cada 28 dias após a pesagem dos animais, e o volumoso fornecido a vontade. $\mathrm{O}$ concentrado foi à base de farelo de soja, milho, uréia, calcário e sal branco. A dieta base (silagem de sorgo) foi calculada para que tivesse em torno de $12 \%$ de proteína bruta (PB) e $70 \%$ de nutrientes digestíveis totais (NDT). Os tratamentos com cana-de-açúcarreceberamomesmoconcentrado da dieta base, esperando-se que a dieta com 1,2\% de concentrado proporcionasse os mesmos teores de PB e NDT da dieta base, e a dieta com 1,0\% de concentrado apresentasse teores inferiores, sem, contudo comprometer o desempenho dos animais.

Os animais foram alimentados duas vezes ao dia (09:00 e 15:00 h) e o consumo de alimentos foi registrado diariamente através da pesagem da quantidade fornecida e das sobras do dia anterior. Foram determinados os teores de matéria seca (MS), cinzas para cálculo da matéria orgânica (MO) e proteína bruta segundo as metodologias descritas por ASSOCIATION OF OFFICIALANALYTICAL CHEMISTS (1990), fibra em detergente neutro de acordo com Van Soest (1994) e o NDT calculado de acordo com Undersander, Mertens e Thiex (1993) e Kearl (1982).

A composição química estimada das diferentes rações está contida na Tabela 1. 
Tabela 1. Composição química das rações utilizadas nos diferentes tratamentos, com base na matéria seca.

\begin{tabular}{lccc}
\hline & \multicolumn{3}{c}{ Tratamento } \\
\cline { 2 - 4 } Componentes (\%) & $\begin{array}{c}\text { Silagem de sorgo + 1,0 } \\
\text { \% concentrado }\end{array}$ & $\begin{array}{c}\text { Cana-de-açúcar + 1,0 } \\
\text { \% concentrado }\end{array}$ & $\begin{array}{c}\text { Cana-de-açúcar + 1,2 } \\
\text { \% concentrado }\end{array}$ \\
\hline Matéria seca & 43,41 & 48,18 & 53,67 \\
Matéria orgânica & 96,82 & 98,55 & 98,71 \\
Proteína bruta & 12,51 & 10,96 & 12,87 \\
Fibra em detergente neutro & 38,00 & 28,85 & 24,28 \\
Nutrientes digestíveis totais & 71,99 & 72,64 & 75,72 \\
\hline
\end{tabular}

A duração do confinamento foi de 100 dias, após o período de 15 dias de adaptação. Foram realizadas quatro avaliações visuais sendo cada uma com duração de 24 horas, e intervalos entre avaliações de quinze dias, com a primeira avaliação realizada após 20 dias do início do confinamento. Foram avaliadas cinco escalas diferentes de intervalos entre as observações: cinco, 10, 15, 20 e 30 minutos. A coleta de dados foi realizada por quatro observadores treinados, divididos em duas duplas que se alternavam a cada período de seis horas. $\mathrm{Na}$ observação noturna dos animais foi utilizada luz artificial localizada (lanterna), de forma a minimizar os efeitos da alteração das condições normais.

Foram avaliados os tempos (min) de ingestão (ING), ruminação (RUM) e ócio (OCIO), a porcentagem de tempo que o animal permaneceu ruminando deitado (PRD) e em ócio deitado (POD) e a freqüência (unidade) de ingestão (FING), ruminação (FRUM) e ócio (FOC), sendo a freqüência determinada como o número de períodos (intervalos) de ingestão, ruminação e ócio.

Os dados referentes aos fatores do comportamento ingestivo foram obtidos de acordo com Bürger et al (2000), conforme descrito abaixo:

$$
\begin{gathered}
\mathrm{EAL}_{\mathrm{MS}}=\mathrm{CMS} / \mathrm{TAL} \\
\mathrm{EAL}_{\mathrm{FDN}}=\mathrm{CFDN} / \mathrm{TAL} \\
\mathrm{ERU}_{\mathrm{MS}}=\mathrm{CMS} / \mathrm{TRU} \\
\mathrm{ERU}_{\mathrm{FDN}}=\mathrm{CFDN} / \mathrm{TRU} \\
\mathrm{TMT}=\mathrm{TAL}+\mathrm{TRU}
\end{gathered}
$$

Sendo, $\operatorname{EAL}_{\mathrm{MS}}(\mathrm{g} \mathrm{MS} / \mathrm{h})$ eficiência de alimentação da matéria seca;

CMS (g MS/dia) consumo de matéria seca;

TAL (h/dia) tempo de alimentação;

$\operatorname{EAL}_{\mathrm{FDN}}(\mathrm{g} \mathrm{FDN} / \mathrm{h})$ eficiência de alimentação da fibra em detergente neutro;

CFDN (g FDN/dia) consumo de fibra em detergente neutro;

$\mathrm{ERU}_{\mathrm{MS}}(\mathrm{g} \mathrm{MS} / \mathrm{h})$ eficiência de ruminação da matéria seca;

TRU (h/dia) tempo de ruminação;

$\mathrm{ERU}_{\mathrm{FDN}}(\mathrm{g} \mathrm{FDN} / \mathrm{h})$ eficiência de ruminação da fibra em detergente neutro;

TMT (h/dia) tempo de mastigação total.

Foram determinados também a ingestão de matéria seca (IMS) em kg/dia e de fibra em detergente neutro (IFDN) em kg/dia.

O delineamento experimental utilizado foi o inteiramente casualizado com 12 animais por tratamento. Os dados obtidos foram submetidos à análise de variância e as médias comparadas pelo teste de Tukey a $5 \%$ de probabilidade, utilizando-se o programa SAEG (UNIVERSIDADE FEDERAL DE VIÇOSA, 2001). 


\section{Resultados e Discussão}

Na Tabela 2 estão contidos os tempos de ingestão, ruminação e ócio, a porcentagem de tempo ruminando deitado e em ócio deitado e a freqüência de ingestão, ruminação e ócio nos diferentes intervalos de tempo.

Tabela 2. Médias dos tempos despendidos em ingestão (ING), ruminação (RUM) e ócio (OCIO), porcentagem de tempo ruminando deitado (PRD) e em ócio deitado (POD) e freqüência de ingestão (FING), ruminação (FRUM) e ócio (FOC) nos diferentes intervalos de tempo.

\begin{tabular}{|c|c|c|c|c|c|c|}
\hline \multirow[b]{2}{*}{ Item } & \multicolumn{5}{|c|}{ Intervalos de tempo } & \multirow[b]{2}{*}{ CV (\%) } \\
\hline & $5 \mathrm{~min}$ & $10 \mathrm{~min}$ & $15 \mathrm{~min}$ & $20 \mathrm{~min}$ & $30 \mathrm{~min}$ & \\
\hline ING (min) & $219,68 \pm 30,11$ & $219,43 \pm 35,61$ & $219,00 \pm 29,46$ & $213,00 \pm 37,52$ & $220,29 \pm 33,84$ & 15,33 \\
\hline RUM (min) & $429,11 \pm 43,68$ & $428,36 \pm 42,97$ & $427,93 \pm 43,96$ & $426,00 \pm 44,83$ & $422,14 \pm 41,86$ & 10,19 \\
\hline OCIO (min) & $784,75 \pm 49,74$ & $785,57 \pm 49,53$ & $786,54 \pm 51,60$ & $794,14 \pm 55,78$ & $790,29 \pm 51,80$ & 6,56 \\
\hline PRD (\%) & 91,32 & 91,20 & 91,00 & 91,70 & 90,74 & - \\
\hline POD (\%) & 67,68 & 67,75 & 67,89 & 66,82 & 68,21 & - \\
\hline FING (no/d) & $17,39 \pm 2,50 \mathrm{a}$ & $11,86 \pm 2,12 b$ & $8,74 \pm 1,45 \mathrm{c}$ & $7,27 \pm 1,38 \mathrm{~d}$ & $5,47 \pm 1,05 \mathrm{e}$ & 17,54 \\
\hline $\begin{array}{l}\text { FRUM } \\
(\mathrm{n} \% / \mathrm{d})\end{array}$ & $19,48 \pm 2,32 \mathrm{a}$ & $16,40 \pm 1,88 b$ & $14,73 \pm 1,76 c$ & $13,41 \pm 1,64 d$ & $11,17 \pm 1,21 \mathrm{e}$ & 11,94 \\
\hline FOC $(n \circ / d)$ & $32,47 \pm 2,54 \mathrm{a}$ & $24,89 \pm 1,78 \mathrm{~b}$ & $20,42 \pm 1,61 \mathrm{c}$ & $17,74 \pm 1,51 \mathrm{~d}$ & $14,03 \pm 0,95 \mathrm{e}$ & 8,01 \\
\hline
\end{tabular}

Médias seguidas de letras diferentes na linha, diferem entre si $(\mathrm{P}<0,05)$ pelo teste de Tukey. CV = coeficiente de variação.

Quando se avaliou os tempos de ingestão, ruminação e ócio (Tabela 2), não se observou diferença significativa $(\mathrm{P}>0,05)$ entre as escalas de cinco, 10, 15, 20 e 30 minutos.

Os dados observados nesse experimento concordam com os relatados por Silva et al. (2005) e Silva et al. (2006) nos quais os autores trabalharam, respectivamente, com novilhas $3 / 4$ Holandês x Zebu e bezerros PO Holandês e também não observaram diferença significativa com até 30 minutos de intervalo nas observações para ingestão, ruminação e ócio.

A percentagem de tempo que os animais permaneceram ruminando deitado ficou em torno de $91 \%$ para todas as escalas de tempo avaliadas, indicando não haver diferença entre essas escalas e mostrando uma condição de bem estar dos animais.

A baixa percentagem de tempo em ócio deitado, entre 66,82 e $68,21 \%$, pode ser explicada pelo fato dos animais permanecerem em pé, durante os períodos diurnos, observando os outros animais e as atividades desenvolvidas próximo às instalações do confinamento.
Quando se avaliou a freqüência de ingestão, ruminação e ócio observou-se que todas as escalas de tempo diferiram $(\mathrm{P}<0,05)$, apresentando uma diminuição na freqüência com o aumento do intervalo entre as observações, fato também constatado por Silva et al., (2006). Essa diminuição na freqüência ocorre devido ao fato dos animais intercalarem, várias vezes durante o dia, as atividades de alimentação, ruminação e ócio, e ao se aumentar os intervalos entre as observações, muitas dessas atividades não são registradas. Entretanto, essa diferença é suprimida quando se avalia o tempo despendido com essas atividades.

Na Tabela 3 observa-se as médias de ingestão de matéria seca e de fibra em detergente neutro, o tempo de mastigação total, a eficiência de alimentação da matéria seca e da fibra em detergente neutro e a eficiência de ruminação da matéria seca e da fibra em detergente neutro nos diferentes intervalos de tempo avaliados. 
Tabela 3. Ingestão de matéria seca (IMS) e de fibra em detergente neutro (IFDN), tempo de mastigação total (TMT), eficiência de alimentação da matéria seca $\left(\mathrm{EAL}_{\mathrm{MS}}\right)$ e da fibra em detergente neutro $\left(\mathrm{EAL}_{\mathrm{FDN}}\right)$ e eficiência de ruminação da matéria seca $\left(E R U_{\mathrm{MS}}\right)$ e da fibra em detergente neutro $\left(\mathrm{ERU}_{\mathrm{FDN}}\right)$ nos diferentes intervalos de tempo.

\begin{tabular}{|c|c|c|c|c|c|c|}
\hline \multirow[b]{2}{*}{ Item } & \multicolumn{5}{|c|}{ Intervalos de tempo } & \multirow[b]{2}{*}{ CV (\%) } \\
\hline & $5 \mathrm{~min}$ & $10 \mathrm{~min}$ & $15 \mathrm{~min}$ & $20 \mathrm{~min}$ & $30 \mathrm{~min}$ & \\
\hline IMS (Kg/d) & $10,10 \pm 1,50$ & $10,10 \pm 1,50$ & $10,10 \pm 1,50$ & $10,10 \pm 1,50$ & $10,10 \pm 1,50$ & 14,84 \\
\hline $\operatorname{IFDN~}(\mathrm{Kg} / \mathrm{d})$ & $3,12 \pm 0,96$ & $3,12 \pm 0,96$ & $3,12 \pm 0,96$ & $3,12 \pm 0,96$ & $3,12 \pm 0,96$ & 30,86 \\
\hline TMT (h/d) & $10,81 \pm 0,82$ & $10,80 \pm 0,81$ & $10,78 \pm 0,85$ & $10,65 \pm 0,91$ & $10,71 \pm 0,84$ & 7,89 \\
\hline $\mathrm{EAL}_{\mathrm{MS}}(\mathrm{Kg} / \mathrm{h})$ & $2,81 \pm 0,60$ & $2,84 \pm 0,70$ & $2,82 \pm 0,62$ & $2,94 \pm 0,75$ & $2,81 \pm 0,59$ & 23,02 \\
\hline $\begin{array}{l}\mathrm{EAL}_{\mathrm{FDN}} \\
(\mathrm{Kg} / \mathrm{h})\end{array}$ & $0,87 \pm 0,31$ & $0,89 \pm 0,35$ & $0,87 \pm 0,31$ & $0,92 \pm 0,37$ & $0,87 \pm 0,30$ & 37,18 \\
\hline $\mathrm{ERU}_{\mathrm{MS}}(\mathrm{Kg} / \mathrm{h})$ & $1,42 \pm 0,21$ & $1,42 \pm 0,20$ & $1,42 \pm 0,21$ & $1,43 \pm 0,19$ & $1,44 \pm 0,22$ & 14,62 \\
\hline $\begin{array}{l}\mathrm{ERU}_{\mathrm{FDN}} \\
(\mathrm{Kg} / \mathrm{h})\end{array}$ & $0,44 \pm 0,13$ & $0,44 \pm 0,12$ & $0,44 \pm 0,12$ & $0,44 \pm 0,12$ & $0,44 \pm 0,13$ & 28,49 \\
\hline
\end{tabular}

$\mathrm{CV}=$ coeficiente de variação. Médias seguidas de letras diferentes na linha, diferem entre si $(\mathrm{P}<0,05)$ pelo teste de Tukey

Não houve diferença significativa entre as variáveis analisadas para os intervalos de tempo de cinco, 10, 15, 20 e 30 minutos, fato também observado por Silva et al. (2006) que avaliou EAL, ERU, TMT e número de bolos ruminais em bezerros holandeses alimentados com sucedâneo e concentrado à base de farelo de soja e milho moído e não observaram diferenças até o intervalo de observação de 30 minutos.

\section{Conclusões}

$\mathrm{O}$ estudo do comportamento ingestivo de ruminantes em confinamento pode ser efetuado com intervalos de até 30 minutos quando se avaliam tempos de ingestão, ruminação e ócio, ingestão de matéria seca e de fibra em detergente neutro, tempo de mastigação total e eficiência de alimentação e ruminação.

Intervalos de 30 minutos permitem que um maior número de animais possa ser observado, sem necessidade de aumentar a mão-de-obra e sem prejuízo da confiabilidade nos resultados.

Quando se avalia a freqüência de ingestão, ruminação e ócio, os intervalos entre as observações devem ser de 5 minutos, uma vez que intervalos maiores subestimam os resultados.

\section{Referências}

ASSOCIATION OF OFFICIAL ANALYTICAL CHEMISTS - AOAC. Official Methods of Analysis. 15. ed. Arlington: AOAC, 1990. v. 1.

BÜRGER, P. J.; PEREIRA, J. C.; QUEIROZ, A. C.; SILVA, J. F. C.; VALADARES FILHO, S. C.; CECON, P. R.; CASALI, A. D. P. Comportamento ingestivo em bezerros holandeses alimentados com dietas contendo diferentes níveis de concentrado. Revista Brasileira de Zootecnia, Viçosa, v. 29. n. 1. p. 236-242, 2000.

DAMASCENO, J. C.; BACCARI JÚNIOR, F.; TARGA, L. A. Respostas comportamentais de vacas holandesas, com acesso à sombra constante ou limitada. Pesquisa Agropecuária Brasileira, Brasília, v. 34, n. 4, p. 709-715, abr. 1999.

KEARL, L. C. Nutrient requirements of ruminants in developing countries. Logan: International Feedstuffs Institute, Utah State University, 1982.

SILVA, R. R.; SILVA, F. F.; CARVALHO, G. G. P.; VELOSO, C. M.; FRANCO, I. L.; AGUIAR, M. S. M. A., CHAVES, M. A.; CARDOSO, C. P.; SILVA, R. R. Avaliação do comportamento ingestivo de novilhas $3 / 4$ Holandês x Zebu alimentadas com silagem de capimelefante acrescida de $10 \%$ de farelo de mandioca: aspectos metodológicos. Ciência Animal Brasileira, Goiânia, v. 6, n. 3, p. 173-177, jul./set. 2005.

SILVA, R. R.; SILVA, F. F.; PRADO, I. N.; CARVALHO, G. G. P.; FRANCO, I. L.; ALMEIDA, V. S.; CARDOSO, C. P.; RIBEIRO, M. H. S. Comportamento ingestivo de bovinos: aspectos metodológicos. Archivos de Zootecnia, Córdoba, v. 55, n. 211, p. 293-296, 2006. 
UNDERSANDER, D.; MERTENS, D. R.; THIEX, N. UNIVERSIDADE FEDERAL DE VIÇOSA. SAEG: Forage analysis procedures. Omaha: National Testing Association, 1993.

Sistema de análises estatísticas e genéticas: manual do usuário. Versão 7.1. Viçosa: Ed. da UFV, 2001.

VAN SOEST, P. J. Nutritional ecology of the ruminant. 2.ed. Ithaca: Cornell University Press, 1994. 